Original

\title{
Immunohistochemical detection of p53 and PCNA in ameloblastoma and adenomatoid odontogenic tumor
}

\author{
Jahanshah Salehinejad ${ }^{1)}$, Reza Zare-Mahmoodabadi ${ }^{1)}$, Shadi Saghafi ${ }^{1)}$, \\ Amir-Hossien Jafarian ${ }^{2)}$, Narges Ghazi ${ }^{1}$, Ali-Reza Rajaei ${ }^{1)}$ and Parviz Marouzi ${ }^{3)}$ \\ ${ }^{1)}$ Department of Oral and Maxillofacial Pathology, Dental Research Center, Faculty of Dentistry, \\ Mashhad University of Medical Sciences, Mashhad, Iran \\ ${ }^{2)}$ Department of Pathology, Ghaem Hospital, Mashhad University of Medical Sciences, Mashhad, Iran \\ ${ }^{3)}$ Department of Social Medicine, Mashhad University of Medical Sciences, Mashhad, Iran
}

(Received 4 November 2010 and accepted 20 April 2011)

\begin{abstract}
Although ameloblastoma and adenomatoid odontogenic tumor (AOT) belong to the same group according to the World Health Organization, they show different biologic behaviors. PCNA, an amplifier of cell proliferation, and p53, a tumor suppressor protein, are overexpressed in some odontogenic lesions. The purpose of this study was to immunohistochemically evaluate the expression of p53 and PCNA to clarify the possible role of these proteins in different behaviors of ameloblastoma and AOT. The immunohistochemical expression of PCNA and p53 was determined in $\mathbf{3 0}$ solid ameloblastomas and $\mathbf{1 2}$ AOTs. Statistical tests including one-way ANOVA, $t$ test, chi-square, Mann-Whitney $U$ and Kendall were used to analyze the data. All tissue sections (except one specimen of plexiform ameloblastoma) exhibited immunoexpression for p53. PCNA was expressed in all specimens. There was no significant difference in PCNA expression between ameloblastomas and AOTs $(P>$ $0.05)$. For $\mathrm{p53}$, there was no statistical difference between subtypes of ameloblastomas $(P>0.05)$, whereas statistical differences were observed between ameloblastomas and AOTs $(P<0.001)$. There was no statistical difference in PCNA intensity of staining
\end{abstract}

Correspondence to Dr. Shadi Saghafi, Department of Oral and Maxillofacial Pathology, Dental Research Center, Faculty of Dentistry, Mashhad University of Medical Sciences, Vakilabad, Blv., Mashhad, P.O. Box 911735-984, Iran

Tel: +98-511-8829501

Fax: +98-511-8829500

E-mail: Saghafis@mums.ac.ir between ameloblastomas and AOTs $(P>0.05)$, whereas the $\mathrm{p53}$ intensity in ameloblastomas was stronger than AOTs $(P<0.05)$. Positive correlation between PCNA and p53 was observed. We concluded that PCNA overexpression is not responsible for the difference in clinical behavior of these two lesions, whereas the expression of p53 in ameloblastoma may explain the more aggressive nature of this tumor compared with AOT. (J Oral Sci 53, 213-217, 2011)

Keywords: ameloblastoma; adenomatoid odontogenic tumor; PCNA; p53; immunohistochemistry.

\section{Introduction}

Odontogenic tumors comprise a complex group of lesions with diverse histopathologic types and clinical behaviors. These lesions rarely exhibit malignant behavior and some of them may represent tumor-like malformations (hamartomas) (1). Ameloblastoma is the most common clinically significant odontogenic tumor of the jaws, accounting for approximately $1 \%$ of all oral tumors $(2,3)$. Although conventional solid ameloblastoma is histologically benign, it has a high rate of recurrence and is locally aggressive (3). From a histologic standpoint, the conventional ameloblastoma shows variable subtypes but these patterns have little bearing on the behavior of the tumor (1). The most common subtypes are follicular and plexiform. Acanthomatous, granular cell, desmoplastic and basal cell are the less common histopathologic patterns $(1,4)$. The adenomatoid odontogenic tumor (AOT) 
represents 3-7\% of all odontogenic tumors. It was once considered as a variant of ameloblastoma, but the clinical features and biologic behavior of AOT show that it is a separate entity (5). This tumor is not aggressive, and recurrence after enucleation is rare (1).

p53 is an inhibitor of cellular growth and its mutant has a role in tumor formation $(6,7)$. p53 gene, a tumor suppressor gene, is located on chromosome 17P13.1. This protein activates genes involved in cell cycle arrest, such as p21 (8-13). Proliferating cell nuclear antigen (PCNA), also known as cyclin, is a useful marker of cellular proliferation and DNA replication. It is synthesized mainly during the G1/S phase of the cell cycle and an increased expression of this protein indicates tumorigenesis $(14,15)$.

The purpose of this study was to evaluate the proliferative activity of ameloblastoma and AOT using PCNA and p53 to clarify the possible role of these proteins in different biological behaviors of these two lesions.

\section{Materials and Methods}

Thirty cases of intraosseous solid ameloblastomas and 12 intraosseous AOTs were retrieved from the records of the Oral and Maxillofacial Pathology Department of Mashhad Dental School, Iran.

Tissue sections stained with hematoxylin-eosin (H\&E) were reviewed by two pathologists and the lesions were grouped as follows: plexiform ameloblastoma $(n=15)$, follicular ameloblastoma $(n=12)$, acanthomatous ameloblastoma $(n=3)$ and AOT $(n=12)$. For immunohistochemical staining, $4-\mu \mathrm{m}$ sections were cut from paraffin-embedded tissue that had been fixed in $10 \%$ buffered formalin and then mounted on Poly-L-Lysin coated glass slides. Sections were deparaffinized in xylene, rehydrated in a decreasing ethanol series and immersed in methanol with $0.3 \%$ hydrogen peroxide for $30 \mathrm{~min}$ in order to block endogenous peroxidase activity. Sections were then washed in phosphate-buffered saline (PBS), incubated with normal serum $(1: 10,10 \mathrm{~min})$ and then treated with primary antibody for $10 \mathrm{~min}$, according to the procedure outlined by the manufacturer. The applied antibodies were anti-p53 (mouse monoclonal antibody, clone DO-7, DAKO, CA, USA) and anti-PCNA (mouse monoclonal antibody, clone PC10, DAKO). After washing with PBS solution, the sections were incubated with polymer-solution. Then, they were washed and reacted with diaminobenzene (DAB) for $5 \mathrm{~min}$, rinsed in distilled water, resulting in a brown reaction.

The positive control was colon adenocarcinoma for p53 and lymphoma for PCNA. As negative control, the primary antibody was not applied. All slides were separately observed by two pathologists under a light microscope with $\times 400$ magnification. In order to assess the activity of PCNA and p53, the number of positive cells, epithelial cells with brown-stained nuclei, among at least 1000 cells were counted in the region of highest activity (hot spot) and the results were multiplied by 100 [number of PCNA- and p53positive cells $/ 1000$ cells $\times 100$ ]. Therefore, the results were expressed as the percentage of total counted cells (index of positivity = IP) (15). In the positive cells, the intensity of staining was graded as weak, moderate and strong. The immunoreactivity of these markers was evaluated in ameloblast-like and stellate reticulum-like cells separately.

The data was statistically analyzed by one-way ANOVA, $t$-test, Kruskal-Wallis, Kendall test and Mann-Whitney $U$ - test using SPSS 15 software. Statistical significance was set at $P<0.05$.

\section{Results}

The 30 cases of ameloblastoma included 16 men and 14 women. The mean age of patients was $33.70 \pm 16.78$ years and all tumors occurred in the mandible. The 12 cases of AOT included 2 men and 10 women with a mean age of $19.08 \pm 6.87$ years. Five cases were in the mandible and 7 in the maxilla. All specimens (except one specimen of plexiform ameloblastoma) showed immunoexpression for p53 and PCNA (Figs. 1, 2). The mean index of positivity (IP) for PCNA was $71.07 \pm 17.90$ in ameloblastoma and $64 \pm 16.17$ in AOT. According to $t$-test, no significant difference was observed in PCNA expression between ameloblastoma and AOT. The mean IP for p53 was 43.97 \pm 15.01 in ameloblastoma and $6.75 \pm 10.08$ in AOT and a statistically significant difference was detected for p53 expression between these two groups $(P<0.001)$ (Table 1). Figure 3 shows the mean PCNA and p53 IP in the studied groups.

According to Table 2, the highest mean IP for PCNA was in acanthomatous ameloblastoma $(76.67 \pm 20.55)$ and the lowest was in AOT $(64 \pm 16.17)$. One-way ANOVA did not confirm a significant difference in PCNA expression among the studied groups and also among the sub-groups of ameloblastomas. The highest and lowest mean IPs for p53 were seen in acanthomatous ameloblastoma (52.67 \pm 13.56) and AOT (16.75 \pm 10.08$)$, respectively. Tukey's test revealed significant differences in $\mathrm{p} 53$ expression among the studied groups $(P<0.001)$, whereas there was no significant difference between subtypes of ameloblastomas $(P=0.589)$ (Table 2). For the PCNA- and p53-positive sections, the staining intensity was graded as weak, moderate and strong. Ameloblastoma showed stronger intensity for p53 than AOT and Mann-Whitney $U$-test demonstrated statistical significance between AOT and ameloblastoma for intensity of p53 staining $(P=0.040)$. 


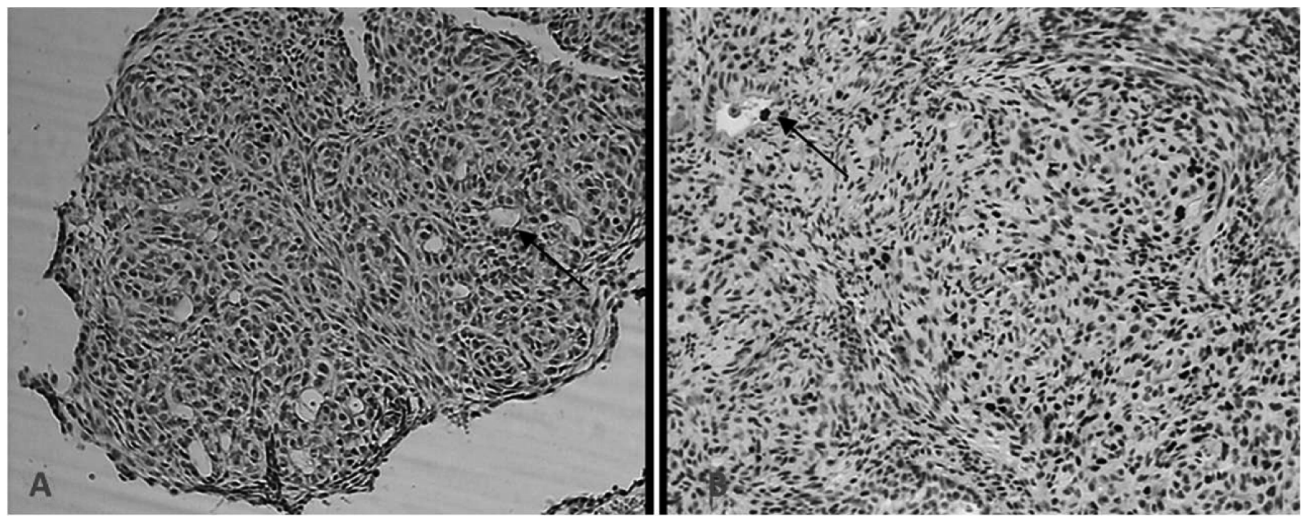

Fig. 1 p53 (A) and PCNA (B) immunoreactivity in AOT. Arrows show duct-like areas (original magnification $\times 100)$.
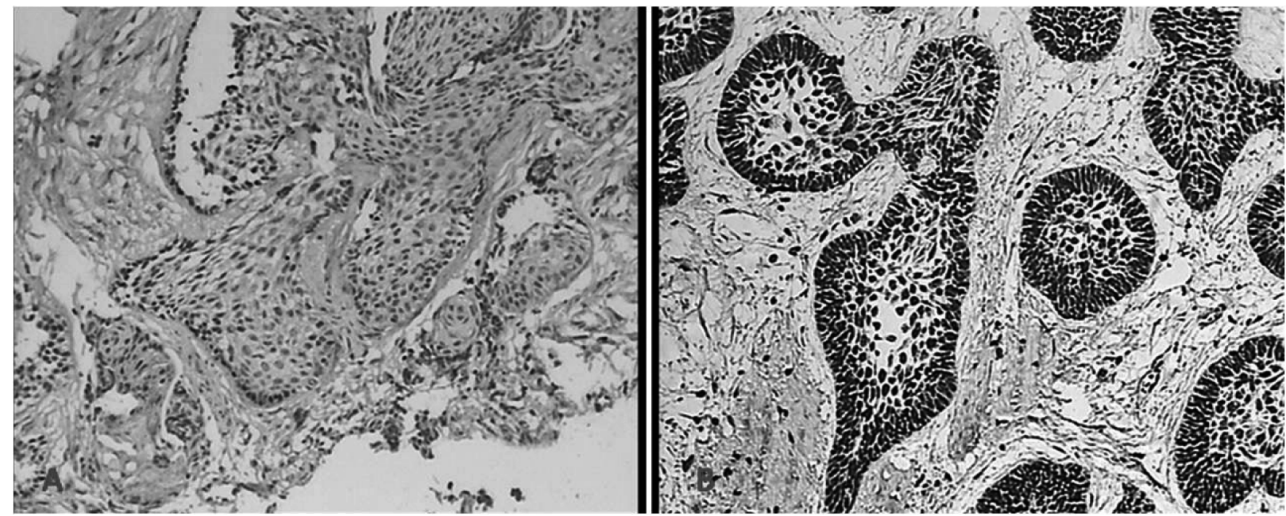

Fig. 2 p53 (A) and PCNA (B) immunoreactivity in follicular ameloblastoma (original magnification $\times 100)$.

Table 1 Index of positivity (IP) for PCNA and p53 in Ameloblastoma and AOT

\begin{tabular}{lllllrl}
\hline Markers & Lesions & Number & Minimum & Maximum & mean IP \pm SD & $P$ value \\
\hline \multirow{2}{*}{ PCNA } & Ameloblastoma & 30 & 25 & 97 & $71.07 \pm 17.90$ & \multirow{2}{*}{0.228} \\
& AOT & 12 & 30 & 93 & $64 \pm 16.17$ & \\
\multirow{2}{*}{ P53 } & Ameloblastoma & 30 & 0 & 65 & $43.97 \pm 15.01$ & \multirow{2}{*}{0.000} \\
& AOT & 12 & 3 & 36 & $16.75 \pm 10.08$ & \\
\hline
\end{tabular}

There was no significant difference in the intensity for PCNA staining between ameloblastoma and AOT when compared by Mann-Whitney $U$ - test $(P=0.348)$.

In the present study, $76 \%$ of p53-positive cells and $80 \%$ of PCNA-positive cells were in the ameloblast-like area. According to Kendall test, a positive correlation between p53 and PCNA was observed.

\section{Discussion}

Ameloblastoma is clinically the most common odontogenic tumor of the jaws, which represents approximately
13 to $24 \%$ of odontogenic tumors, whereas AOT is a rather uncommon tumor of odontogenic origin $(2,3)$. Although both ameloblastoma and AOT are classified by the World Health Organization as epithelial odontogenic tumors, they show different biologic behaviors. Ameloblastoma is a potentially destructive tumor, whereas AOT presents slow, progressive, and circumscribed growth. Various factors have been associated with the aggressive behavior of ameloblastomas, such as the increase in the proliferative potential and changes in the expression of tumor suppressor genes and their protein products $(6,9)$. 
Table 2 Index of positivity (IP) for PCNA and p53 in the studied groups

\begin{tabular}{llllllll}
\hline Markers & \multicolumn{1}{c}{ Lesions } & Number & Minimum & Maximum & mean IP \pm SD & $P$ value & $P$ value \\
\hline PCNA & AOT & 12 & 30 & 83 & $64.00 \pm 16.17$ & - \\
& Plexiform Ameloblastoma & 15 & 25 & 93 & $70.40 \pm 20.42$ & 0.654 \\
& Follicular Ameloblastoma & 12 & 35 & 97 & $70.50 \pm 15.11$ & 0.858 \\
& Acanthomatous Ameloblastoma & 3 & 53 & 90 & $76.67 \pm 20.55$ & \\
P53 & 12 & 3 & 36 & $16.75 \pm 10.08$ & - \\
& AOT & 15 & 0 & 60 & $42.60 \pm 15.72$ & \\
& Plexiform Ameloblastoma & 12 & 16 & 63 & $43.50 \pm 14.89$ & 0.859 \\
& Follicular Ameloblastoma & 38 & 65 & $52.67 \pm 13.65$ & 0.000 \\
\hline
\end{tabular}

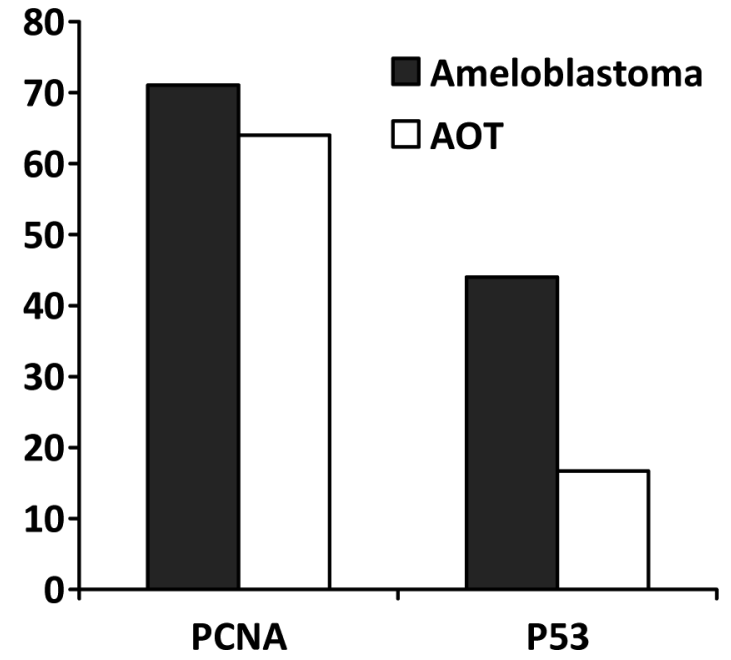

Fig. 3 The mean index of positivity (IP) in the studied lesions.

In the present study, expression of p53 antigen in the ameloblastoma was significantly higher than AOT. Previous research has confirmed the overexpression of p53 in ameloblastoma, which is consistent with our findings (1519). The acanthomatous type of ameloblastoma showed the highest mean IP for p53, but no significant difference was detected in different subtypes of ameloblastoma as described by El-Sissy et al. (19). Barboza et al. (15) indicated that ameloblastoma had higher p53 labeling index than AOT. They observed that the plexiform subtype had the strongest labeling index followed by the follicular subtype, while in our study the highest mean IP for p53 was seen in acanthomatous ameloblastoma. Regezi et al. (20), Moghadam et al. (21) and Migaldi et al. (3) reported that $\mathrm{p} 53$ protein was not enhanced in ameloblastoma, which did not agree with our findings. However, we believe that comparison of our results for p53 staining to those of other studies may not be useful to assess the kinetic cellular events because of variations in the methodology for immunohistochemical staining.

In the present study, the mean index of positivity (IP) for PCNA antigen was higher in ameloblastoma than AOT, with the highest IP being shown for the acanthomatous type. Significant differences were not detected among the studied groups when using one-way ANOVA. Higher PCNA positivity may indicate higher cellular proliferation rate, which would explain the more aggressive nature of the ameloblastoma compared to the AOT. In a study by Barboza (15), there was significantly higher incidence of PCNA labeling in the cases of ameloblastoma than in the cases of AOT which agreed with our findings.

In our study, the peripheral epithelial cells of the nests showed strong PCNA IP, which is in accordance with the studies of Fujita et al. (14). Yamamoto et al. (22) evaluated PCNA expression in AOT, ameloblastic fibroma, odontoameloblastoma and ameloblastic fibro-odontoma. They reported that the PCNA-positive cells were rarely observed in these tumors (22). Funaoka et al. (23) verified the higher PCNA labeling index for follicular ameloblastoma than plexiform type. In another study, the basal cell type showed the highest labeling index (24). Ueno et al. (25) and Reichart et al. (26) also stated that the high expression of PCNA in follicular ameloblastoma can explain its increased potential of recurrence, but in our study, the highest mean IP for PCNA was in acanthomatous ameloblastoma.

In another study, we had concluded that p53 and PCNA antigens were possible parameters for differentiation of calcifying odontogenic cyst subtypes (27). The PCNA and p53 protein immunodetection results of this study suggest that the ameloblastoma has greater proliferative potential than the AOT, which may explain its more aggressive and invasive nature.

\section{Acknowledgments}

This study was supported by a grant (No. 87440) from the Vice Chancellor of Mashhad, University of Medical Sciences, Iran.

\section{References}

1. Neville BW, Damm DD, Allen CM, Bouquot JE (2009) Oral and maxillofacial pathology. 3rd ed, 
Saunders, Philadelphia, 701-715.

2. Nodit L, Barnes L, Childers E, Finkelstein S, Swalsky P, Hunt J (2004) Allelic loss of tumor suppressor genes in ameloblastic tumors. Mod Pathol 17, 1062-1067.

3. Migaldi M, Sartori G, Rossi G, Cittadini A, Sgambato A (2008) Tumor cell proliferation and microsatellite alterations in human ameloblastoma. Oral Oncol 44, 50-60.

4. Cawson RA, Binne WH, Barrett AW, Wright JM (2001) Oral disease: clinical and pathological correlations. 3rd ed, Mosby, London, 52-68.

5. Gnepp DR (2008) Diagnostic surgical pathology of the head and neck. 2nd ed, Saunders, Philadelphia, 616-623.

6. Sandra F, Nakamura N, Kanematsu T, Hirata M, Ohishi M (2002) The role of MDM2 in the proliferative activity of ameloblastoma. Oral Oncol 38, 153-157.

7. Finlay CA, Hinds PW, Levine AJ (1989) The p53 proto-oncogene can act as a suppressor of transformation. Cell 57, 1083-1093.

8. Kumar V, Cotran RS, Robbins SL (2003) Robbins basic pathology. 7th ed, Saunders, Philadelphia, 270-304.

9. Kumamoto H, Izutsu T, Ohki K, Takahashi N, Ooya $\mathrm{K}$ (2004) p53 gene status and expression of p53, MDM2, and p14 proteins in ameloblastomas. J Oral Pathol Med 33, 292-299.

10. Nigro JM, Baker SJ, Preisinger AC, Jessup JM, Hostetter R, Cleary K, Bigner SH, Davidson N, Baylin S, Devilee P, Glover T, Collins FS, Weslon A, Modali R, Harris CC, Vogelstein B (1989) Mutation in the p53 gene occur in diverse human tumour types. Nature 342, 705-708.

11. Ko LJ, Prives C (1996) p53: puzzle and paradigm. Genes Dev 10, 1054-1072.

12. Furihata M, Sonobe H, Ohtsuki Y, Yamashita M, Morioka M, Yamamoto A, Terao N, Kuwahara M, Fujisaki N (1996) Detection of p53 and bcl-2 protein in carcinoma of the renal pelvis and ureter including dysplasia. J Pathol 178, 133-139.

13. Levine AJ, Momand J, Finlay CA (1991) The p53 tomour suppressor gene. Nature 351, 453-456.

14. Fujita S, Seki S, Fujiwara M, Ikeda T (2008) Midkine expression correlating with growth activity and tooth morphogenesis in odontogenic tumors. Hum Pathol 39, 694-700.

15. Barboza CA, Pereira Pinto L, Freitas Rde A, Costa Ade L, Souza LB (2005) Proliferating cell nuclear antigen (PCNA) and p53 protein expression in ameloblastoma and adenomatoid odontogenic tumor. Braz Dent J 16, 56-61.

16. Al-Salihi KA, Li LY, Azlina A (2006) p53 gene mutation and protein expression in ameloblastomas. Braz J Oral Sci 5, 1034-1040.

17. Appel T, Gath R, Wernert N, Martini M, Bergé S (2004) Molecular biological and immunohistochemical analysis of tp53 in human ameloblastomas. Mund Kiefer Gesichtschir 8, 167172. (in German)

18. Slootweg PJ (1995) p53 protein and Ki-67 reactivity in epithelial odontogenic lesions. An immunohistochemical study. J Oral Pathol Med 24, 393-397.

19. El-Sissy NA (1999) Immunohistochemical detection of p53 protein in ameloblastoma types. East Mediterr Health J 5, 478-489.

20. Regezi JA, Sciubba JJ, Jordan RCK (2008) Oral pathology: clinical pathologic correlations. 5th ed, Saunders, St Louis, 261-270.

21. Moghadam BKH, Yazdi I, Barker B, Cob C, Cumming CG (1997) Immunohistochemical determination of tumor-associated antigens in ameloblastoma and odontogenic cysts. Acta Med Iran 35, 1-7.

22. Yamamoto K, Yoneda K, Yamamoto T, Ueta E, Osaki T (1995) An immunohistochemical study of odontogenic mixed tumours. Eur J Cancer B Oral Oncol 31B, 122-128.

23. Funaoka K, Arisue M, Kobayashi I, Iizuka T, Kohgo T, Amemiya A, Totsuka Y (1996) Immunohistochemical detection of proliferating cell nuclear antigen (PCNA) in 23 cases of ameloblastoma. Eur J Cancer B Oral Oncol 32B, 328-332.

24. Kumamoto H, Kimi K, Ooya K (2001) Detection of cell cycle-related factors in ameloblastomas. J Oral Pathol Med 30, 309-315.

25. Ueno S, Mushimoto K, Shirasu R (1989) Prognostic evaluation of ameloblastoma based on histologic and radiographic typing. J Oral Maxillofac Surg 47, 1115.

26. Reichart PA, Philipsen HP, Sonner S (1995) Ameloblastoma: biological profile of 3677 cases. Eur J Cancer B Oral Oncol 31B, 86-99.

27. Saghafi S, Zare-Mahmoodabadi R, Salehinejad J, Kadeh H, Afzal-Aghaee M (2010) Immunohistochemical analysis of p53 and PCNA expression in calcifying odontogenic cyst. J Oral Sci 52, 609-613. 